\title{
Sự thật về nội lực Việt Nam trong trích dẫn khoa học hàng đâu thế giới 2021
}

\author{
TS. Lê Văn Út \\ Trưởng nhóm Nhóm nghiên cứu trắc lượng thông tin \\ Trường Đại học Tôn Đức Thắng
}

Báo Điện tử Dân Trí

Ngày 30 tháng 10 năm 2021

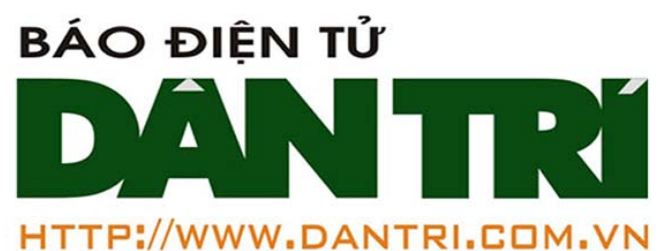

https://dantri.com.vn/giao-duc-huong-nghiep/su-that-ve-noi-luc-viet-namtrong-trich-dan-khoa-hoc-hang-dau-the-gioi-2021-

20211030083557165.htm 


\section{DANTI}

Thứ Bảy 30/10/2021 - 08:37

\section{Sự thật về nội lực Việt Nam trong trích dẫn khoa học hàng đầu thế giới 2021}

\section{(Dân trí) - Thống kê mới nhất, năm 2021 Việt Nam có 65 đại diện nội lục vào top nhà khoa học trích dẫn hàng đầu thế giới.}

Hàng năm, việc công bố danh sách các nhà nghiên cứu thuộc tốp 100 ngàn các nhà nghiên cứu có trích dẫn khoa học hàng đầu thế giới theo Cơ sở dữ liệu Scopus là vấn đề rất được cộng đồng khoa học quan tâm.

Năm 2021, kết quả này đã tiếp tục được công bố bởi nhóm tác giả gồm TS. John P. A. Ioannidis (giáo sư thực thụ về y khoa, nghiên cứu sức khỏe và chính sách, khoa học dữ liệu y sinh và thống kê học thuộc Đại học Stanford, Mỹ), Jeroen Baas (giám đốc dữ liệu của Nhà xuất bản Elsevier, Hà Lan) và TS. Kevin Boyack (giám đốc điều hành của Công ty SciTech Strategies, Mỹ; chuyên về trắc lượng khoa học).

Việc sử dụng Cơ sở dữ liệu Scopus thuộc Nhà xuất bản Elsevier của Hà Lan làm cho kết quả của nhóm tác giả trở nên tin cậy và có ảnh hưởng sâu rộng trong cộng đồng khoa học thế giới bởi lẽ Scopus là một trong hai cơ sở dữ liệu khoa học uy tín nhất trên thế giới hiện nay, bên cạnh Cơ sở dữ liệu Web of Science của Mỹ.

Trong năm nay, nhóm tác giả trên công bố các danh sách các nhà nghiên cứu có trích dẫn khoa học cao thuộc tốp 100.000 hoặc tốp 2\% trên toàn thế giới gồm xét theo thành tựu trọn đời, thành tựu năm gần nhất và thành tựu theo chuyên ngành.

Các tiêu chí dùng để đánh giá đẳng cấp trích dẫn khoa học của một nhà nghiên cứu gồm tổng số trích dẫn được chuẩn hóa, chỉ số H (H-index), chỉ số đồng tác giả (HMindex), chỉ số trích dẫn theo vị trí tác giả, chỉ số tự trích dẫn và không tự trích dẫn.

Như vậy, có thể thấy đẳng cấp trích dẫn khoa học được xem xét khá toàn diện, không phải thuần túy là tổng số trích dẫn khoa học mà một nhà nghiên cứu có được.

Trong bài viết này, hai danh sách được quan tâm là danh sách tốp 100.000 hoặc tốp $2 \%$ các nhà nghiên cứu có trích dẫn khoa học xét theo thành tựu trọn đời (career, tất cả các kết quả trích dẫn khoa học đến năm gần nhất) và theo năm (single year, kết quả trích dẫn khoa học của một năm gần nhất).

Trên tinh thần phát triển nội lực, bài viết chỉ quan tâm đến các nhà nghiên cứu có quốc tịch Việt Nam và đang là nhân sự cơ hữu thuộc các tổ chức thuộc Việt Nam (gọi tắt: nhà nghiên cứu nội lực).

Những nhà nghiên cứu có quốc tịch Việt Nam nhưng được liệt kê dưới tên đơn vị kiêm nhiệm thì không được xem xét.

Dù có sự giới hạn khá hẹp (nhưng đúng nghĩa nội lực) so với các kết quả thống kê gần đây nhưng kết quả trong bài này cho thấy có sự tăng mạnh những nhà nghiên cứu của Việt Nam trong tốp 100.000 hoặc tốp $2 \%$ các nhà nghiên cứu có trích dẫn khoa học cao 
trên toàn thế giới.

Cụ thể, trong năm 2021 Việt Nam có tổng cộng 22 nhà nghiên cứu được liệt kê trong tốp 100.000 hoặc tốp $2 \%$ nhà nghiên cứu có trích dẫn khoa học hàng đầu thế giới tính trên thành tựu trọn đời, và $\mathbf{6 5}$ người tương ứng tính trên thành tựu năm gần nhất.

Kết quả này là một sự đột phá rất đáng kể về sự nhận diện thông qua trích dẫn khoa học của các công trình nghiên cứu từ các nhà nghiên cứu nội lực của Việt Nam; bởi lẽ năm 2019 Việt Nam không có nhà nghiên cứu nội lực nào của Việt Nam được vào danh sách thành tựu trọn đời và chỉ có $\mathbf{1 0}$ người được vào danh sách thành tựu năm.

Bằng các phương pháp thống kê dữ liệu, phân tích dữ liệu thu thập từ nhóm tác giả trên, đối chiếu với dữ liệu gốc từ Cở sở dữ liệu Scopus và Web of Science và đối chiếu lực lượng nhân sự nội lực của các viện/đại học (trong đó có gọi điện trực tiếp để kiểm tra thông tin), các kết quả thu được như sau:

Thư nhất, Đối với thành tựu trọng đời, Việt Nam có tổng cộng 22 nhà nghiên cứu nội lực được liệt kê trong tốp 100.000 nhà nghiên cứu có trích dẫn khoa học hàng đầu thế giới từ dữ liệu gốc gồm:

\begin{tabular}{|l|l|r|r|r|}
\hline \multicolumn{1}{|c|}{ authfull } & \multicolumn{1}{|c}{ inst_name } & np6020 & firstyr & lastyr \\
\hline Nguyen-Xuan, H. & Đại học Công nghệ Thành phố Hồ Chi & 219 & 2008 & 2021 \\
\hline Duc, Nguyen Dinh & Vietnam National University Hanoi & 140 & 1996 & 2021 \\
\hline $\begin{array}{l}\text { Tuy, Hoang } \\
\text { Son, Le Hoang }\end{array}$ & Hanoi Institute of Mathematics & 111 & 1971 & 2018 \\
\hline Khoa, Dao T. & Vietnam National University Hanoi & 180 & 2010 & 2021 \\
\hline Van Hung, Pham & Vietnam Atomic Energy Institute & 94 & 1985 & 2021 \\
\hline Phat, Vu N. & International University,Vietnam Na & 53 & 2004 & 2021 \\
\hline Van Hieu, Nguyen & Vietnam Academy of Science and Te & 125 & 1988 & 2021 \\
\hline An, Nguyen Ba & Phenikaa University & 142 & 2004 & 2021 \\
\hline Nam, Nguyen Hai & Vietnam Academy ot Science and Te & 94 & 1981 & 2021 \\
\hline Tran, Hai Nguyen & Hanoi College of Pharmacy & 84 & 1999 & 2021 \\
\hline Bui, Hung & Duy Tan University & 67 & 2016 & 2021 \\
\hline Hoang, Nhat Duc & VinAl Research & 86 & 1995 & 2021 \\
\hline Pham, Binh Thai & Duy Tan University & 82 & 2012 & 2021 \\
\hline Hoang, Anh Tuan & University of Transport Technology & 93 & 2016 & 2021 \\
\hline Nam, Nguyen Dang & Đại học Công nghệ Thành phố Hồ Chí & 56 & 2017 & 2021 \\
\hline Nguyen, Trung Kien & Duy Tan University & 118 & 2008 & 2021 \\
\hline Hien, Le Van & University of Transport and Commun & 52 & 2007 & 2021 \\
\hline Dao, Van Duong & Hanoi National University of Educatiı & 61 & 2005 & 2021 \\
\hline Thai, Chien H. & Phenikaa University & 96 & 2011 & 2021 \\
\hline Hoang, Vo Van & Ton-Duc-Thang University & 61 & 2010 & 2021 \\
\hline Vuong, Quan Hoang & Đại học Công nghệ Thành phố Hồ Chí & 61 & 1996 & 2021 \\
\hline
\end{tabular}

Thông tin chi tiết được xếp theo thứ tụ hạng giảm dần nhu sau:

Nhà nghiên cứu Đơn vị

Nguyễn Xuân Hùng ĐH Công nghệ TP.HCM

Nguyễn Đình Đức ĐHQG Hà Nội

\section{Hạng thế giới}

48861

60916 
Hoàng Tụy

Viện Hàn lâm KH\&CN Việt Nam

81258

Lê Hoàng Sơn

ĐHQG Hà Nội

84000

Đào Tiến Khoa

Viện năng lượng nguyên tử Việt Nam 111448

Phạm Văn Hùng

ĐHQG TP.HCM

123588

Vũ Ngọc Phát

Viện Hàn lâm KH\&CN Việt Nam

125169

Nguyễn Văn Hiếu

ĐH Phenikaa

125835

Nguyễn Bá Ân Viện Hàn lâm KH\&CN Việt Nam

126306

Nguyễn Hải Nam

ĐH Dược Hà Nội

128490

Trần Nguyễn Hải

ĐH Duy Tân

150613

Bùi Hải Hưng

Viện nghiên cứu AI - Vingroup

154787

Hoàng Nhật Đức ĐH Duy Tân

162525

Phạm Thái Bình ĐH Công nghệ Giao thông Vận tải

162649

Hoàng Anh Tuấn ĐH Công nghệ TP.HCM

165272

Nguyễn Đăng Nam ĐH Duy Tân

167821

Nguyễn Trung Kiên ĐH Giao thông Vận tải TP.HCM

169296

Lê Văn Hiện

ĐHSP Hà Nội

171699

Đào Văn Dương ĐH Phenikaa

174338

Thái Hoàng Chiến ĐH Tôn Đức Thắng

175372

Võ Văn Hoàng

ĐHQG TP.HCM

182749

Vương Quân Hoàng ĐH Phenikaa

185537

Kết quả cho thấy có 13 tổ chức có nhà nghiên cứu nội lực có trích dẫn cao gồm 10 đại học và 3 viện nghiên cứu; trong đó có 1 viện nghiên cứu ngoài công lập và 2 đại học ngoài công lập; cụ thể là Viện Hàn lâm KH\&CN Việt Nam, ĐHQG TP.HCM, ĐHQG Hà Nội, ĐHSP Hà Nội, ĐH Tôn Đức Thắng, ĐH Công nghệ Giao thông Vận tải, ĐH 
Giao thông Vận tải TP.HCM, ĐH Dược Hà Nội, Viện năng lượng nguyên tử Việt Nam, ĐH Duy Tân, ĐH Phenikaa, ĐH Công nghệ TP.HCM và Viện nghiên cứu $\mathrm{AI}$ Vingroup.

Điều rất đáng quan tâm là cả hai đại học ngoài công lập là ĐH Phenikaa và ĐH Duy Tân là có nhiều nhà nghiên cứu nhất ( 3 người), cùng với Viện hàn lâm khoa học và công nghệ Việt Nam (cũng có 3 người).

Có thể nói, những nhà nghiên cứu nội lực của Việt Nam được vào danh sách thành tựu trọn đời là những người có đẳng cấp cao về công bố và trích dẫn khoa học, kết quả này mang tính bền vững và ổn định hơn.

Cũng xin lưu ý thêm, trong danh sách thành tựu trọn đời của Việt Nam, có một nhà khoa học đã mất hai năm trước, Cố GS. Hoàng Tụy, Cố Viện trưởng Viện toán học Việt Nam. Công trình Scopus gần nhất của ông được công bố vào năm 2018.

Điều này thật sự rất thú vị vì các nhà nghiên cứu có thể không còn nữa nhưng những công trình của họ vẫn được sự quan tâm của cộng đồng khoa học trên toàn thế giới.

Đây có thể là động lực để các nhà nghiên cứu chân chính dấn thân không mệt mỏi vào nghiệp nghiên cứu.

Từ đó, họ có thể để lại những công trình có ý nghĩa quan trọng cho chuyên ngành và có thể cho những ứng dụng góp phần cải tiến chất lượng cuộc sống của nhân loại. Và khi đó, nhà nghiên cứu có thể tồn tại mãi mãi dù họ có thể không còn nữa.

Thư hai, Đối với thành tựu năm gần nhất, Việt Nam có tổng cộng $\mathbf{6 5}$ nhà nghiên cứu nội lực được liệt kê trong tốp 100.000 hoặc tốp 2\% nhà nghiên cứu có trích dẫn khoa học hàng đầu thế giới từ dữ liệu gốc gồm:

\begin{tabular}{|c|c|c|c|c|}
\hline authfull & inst_name & np6020 & firstyr & lastyr \\
\hline Duc, Nguyen Dinh & Vietnam National University Hanoi & 140 & 1996 & 2021 \\
\hline Son, Le Hoang & Vietnam National University Hanoi & 180 & 2010 & 2021 \\
\hline Nguyen-Xuan, $\mathrm{H}$. & Đại học Công nghệ Thành phố Hồ Chí N & 219 & 2008 & 2021 \\
\hline Vo, Xuan Vinh & University of Economics Ho Chi Minh C & 90 & 2005 & 2021 \\
\hline Tran, Hai Nguyen & Duy Tan University & 67 & 2016 & 2021 \\
\hline Tran, Bach Xuan & Hanoi Medical University & 254 & 2015 & 2021 \\
\hline Pham, Binh Thai & University of Transport Technology & 93 & 2016 & 2021 \\
\hline Hoang, Nhat Duc & Duy Tan University & 82 & 2012 & 2021 \\
\hline van Hieu, Dang & Thang Long University & 49 & 2014 & 2021 \\
\hline Hoang, Anh Tuan & Đại học Công nghệ Thành phố Hồ Chí $N$ & 56 & 2017 & 2021 \\
\hline Van Hung, Pham & International University, Vietnam Nati & 53 & 2004 & 2021 \\
\hline Nguyen-Thoi, T. & Ton-Duc-Thang University & 185 & 2007 & 2021 \\
\hline Trung, Tran & Hoa Binh University & 72 & 2005 & 2021 \\
\hline Thai, Chien H. & Ton-Duc-Thang University & 61 & 2010 & 2021 \\
\hline Nguyen, Trung Kien & University of Transport and Communic & 52 & 2007 & 2021 \\
\hline Nguyen, Truong Khang & Ton-Duc-Thang University & 145 & 2007 & 2021 \\
\hline Nguyen, Thang Trung & Ton-Duc-Thang University & 72 & 2014 & 2021 \\
\hline Le, Thai Ha & Fulbright University Vietnam & 33 & 2012 & 2021 \\
\hline Nam, Nguyen Dang & Duy Tan University & 118 & 2008 & 2021 \\
\hline
\end{tabular}




\begin{tabular}{|c|c|c|c|c|}
\hline Van Hieu, Nguyen & Phenikaa University & 142 & 2004 & 2021 \\
\hline Phung-Van, P. & Đại học Công nghệ Thành phố Hồ Chí $N$ & 51 & 2012 & 2021 \\
\hline Nguyen, Hoang Long & Hanoi University of Mining and Geolo६ & 75 & 2018 & 2021 \\
\hline Hieu, Dang Van & Thang Long University & 19 & 2016 & 2021 \\
\hline Dao, Van Duong & Phenikaa University & 96 & 2011 & 2021 \\
\hline Chu, Dinh Toi & Hanoi National University of Educatior & 121 & 2014 & 2021 \\
\hline Vuong, Quan Hoang & Phenikaa University & 96 & 2011 & 2021 \\
\hline Tran, Phong D. & Vietnam Academy of Science and Tecr & 56 & 2009 & 2021 \\
\hline Hien, Le Van & Hanoi National University of Educatior & 61 & 2005 & 2021 \\
\hline Van Le, Quyet & Duy Tan University & 122 & 2014 & 2021 \\
\hline Phan, Nam T.S. & Đại học Công nghệ Thành phố Hồ Chí $N$ & 114 & 2004 & 2021 \\
\hline Vo, Dai Viet $\mathrm{N}$. & Nguyen Tat Thanh University & 198 & 2009 & 2021 \\
\hline Hoa, Nguyen Duc & International Training Institute for $\mathrm{Ma}$ & 119 & 2006 & 2021 \\
\hline $\mathrm{Ta}$, Son Xuat & Hanoi University of Science and Techn & 80 & 2010 & 2021 \\
\hline Hoat, D. M. & Duy Tan University & 72 & 2018 & 2021 \\
\hline Nguyen, Quyen & Duy Tan University & 59 & 2014 & 2021 \\
\hline Tuan, Le Anh & Vietnam Maritime University & 26 & 2011 & 2021 \\
\hline Bach, Long Giang & Nguyen Tat Thanh University & 217 & 2012 & 2021 \\
\hline Khoa, Dao T. & Vietnam Atomic Energy Institute & 94 & 1985 & 2021 \\
\hline Phuc, Huynh V. & Dong Thap University & 126 & 2010 & 2021 \\
\hline Ninh, Dinh Gia & Hanoi University of Science and Techn & 20 & 2014 & 2021 \\
\hline Nam, Nguyen Hai & Hanoi College of Pharmacy & 84 & 1999 & 2021 \\
\hline Le, Minh Quy & Hanoi University of Science and Techn & 42 & 2004 & 2021 \\
\hline Phat, Vu N. & Vietnam Academy of Science and Tect & 125 & 1988 & 2021 \\
\hline Pham, Van Viet & Ho Chi Minh City University of Transpc & 25 & 2018 & 2021 \\
\hline Anh, Pham Ngoc & Posts and Telecommunications Institu & 78 & 1981 & 2021 \\
\hline Tung, Hoang Van & Hanoi Architectural University & 16 & 2010 & 2021 \\
\hline Van Do, Vuong Nguyen & Ton-Duc-Thang University & 29 & 2014 & 2020 \\
\hline Tran, Tuan Hiep & Phenikaa University & 53 & 2012 & 2021 \\
\hline Pham, Thanh Dong & Vietnam National University Hanoi & 43 & 2012 & 2021 \\
\hline Tran, Loc Vinh & Ton-Duc-Thang University & 26 & 2011 & 2020 \\
\hline Tuan, Nguyen Huy & Viet Nam National University Ho Chi N & 110 & 2007 & 2021 \\
\hline Nguyen, $\mathrm{C}$. & Le Quy Don Technical University & 71 & 2015 & 2021 \\
\hline Chung, Nguyen Thanh & Quang Binh University & 71 & 2008 & 2021 \\
\hline Nguyen, Thi Hong & Can Tho University & 30 & 2013 & 2021 \\
\hline Hieu, Nguyen $\mathrm{N}$. & Duy Tan University & 145 & 2007 & 2021 \\
\hline Luong, Nguyen Cong & Phenikaa University & 15 & 2016 & 2021 \\
\hline Ly, Hai Bang & University of Transport Technology & 63 & 2014 & 2021 \\
\hline Tran, Huy Hung & Phenikaa University & 48 & 2014 & 2021 \\
\hline Vo, Bay & Đại học Công nghệ Thành phố Hồ Chí N & 162 & 2009 & 2021 \\
\hline Van Tung, Hoang & Hanoi Architectural University & 17 & 2010 & 2021 \\
\hline Do, Hoa Thi & Nguyen Tat Thanh University & 54 & 2015 & 2020 \\
\hline Dao, Thanh Phong & Ton-Duc-Thang University & 55 & 2013 & 2021 \\
\hline Son, Ninh The & Vietnam Academy of Science and Tect & 28 & 2015 & 2021 \\
\hline Nguyen, Tan $\mathrm{N}$. & Ton-Duc-Thang University & 58 & 2016 & 2021 \\
\hline Duc, Nguyen Minh & Children's Hospital 2 & 41 & 2017 & 2021 \\
\hline
\end{tabular}

Thông tin chi tiết được xếp theo thứ tụ hạng giảm dần là: 
Nhà nghiên cứu

Nguyễn Đình Đức

Lê Hoàng Sơn

Nguyễn Xuân Hùng

Võ Xuân Vinh

Trần Nguyễn Hải

Trần Xuân Bách

Phạm Thái Bình

Hoàng Đức Nhật

Đặng Văn Hiếu

Hoàng Anh Tuấn

Phạm Văn Hùng

Nguyễn Thời Trung

Trần Trung

Thái Hoàng Chiến

Nguyễn Trung Kiên

ĐH Giao thông Vận tải TP.HCM

ĐH Tôn Đức Thắng

ĐH Hòa Bình

ĐH Tôn Đức Thắng

Nguyễn Trương Khang ĐH Tôn Đức Thắng

Nguyễn Trung Thắng ĐH Tôn Đức Thắng

Lê Thái Hà

ĐH Fulbright Việt Nam

Nguyễn Đăng Nam Duy Tân

ĐH Phenikaa

Nguyễn Văn Hiếu

\author{
DH Phenikaa
}

Phùng Văn Phúc

ĐH Công nghệ TP.HCM
50677

62836

66151

\section{Hạng thế giới}

5950

6766

6819

9529

14750

19882

21589

22302

31140

32939

37521

46054

48770

53487

74064

81654

82172

83197 
Nguyễn Hoàng Long

ĐH Mỏ - Địa chất Hà Nội

94129

Đặng Văn Hiếu

Đào Văn Dương

Chu Đình Tới

Vương Quân Hoàng

Trần Đình Phong

Lê Văn Hiện

Lê Văn Quyết

Phan Thanh Sơn Nam

Võ Nguyễn Đại Việt

Nguyễn Đức Hòa

Tạ Sơn Xuất

Đỗ Minh Hoạt

Nguyễn Quyên

Lê Anh Tuấn

Bạch Long Giang

Đào Tiến Khoa

Huỳnh Vĩnh Phúc

Nguyễn Thời Trung

Đinh Gia Ninh

Nguyễn Hải Nam

Lê Minh Quý
ĐH Thăng Long

ĐH Phenikaa

108442

ĐHSP Hà Nội

110977

ĐH Phenikaa

115981

Viện Hàn lâm KH\&CN Việt Nam

119201

ĐHSP Hà Nội

121703

ĐH Duy Tân

122357

ĐHQG TP.HCM

124623

ĐH Nguyễn Tất Thành

130351

ĐH Bách khoa Hà Nội

131688

ĐH Bách khoa Hà Nội

133211

ĐH Duy Tân

134747

ĐH Duy Tân

137322

ĐH Hàng Hải Việt Nam

138319

ĐH Nguyễn Tất Thành

138947

Viện năng lượng nguyên tử Việt Nam 140933

ĐH Đồng Tháp

147636

ĐH Tôn Đức Thắng

148476

ĐH Bách khoa Hà Nội

148576

ĐH Dược Hà Nội

150305

ĐH Bách khoa Hà Nội

151654 
Vũ Ngọc Phát

Viện Hàn lâm KH\&CN Việt Nam

Phạm Văn Việt

ĐH Giao thông vận tải TP.HCM

Học viện CNBCVT Việt Nam

Phạm Ngọc Anh

Hoàng Văn Tùng

ĐH Kiến trúc Hà Nội

Đỗ Nguyễn Văn Vương ĐH Tôn Đức Thắng

ĐH Phenikaa

Trần Tuấn Hiệp

Phạm Thanh Đồng

ĐHQG Hà Nội

Trần Vĩnh Lộc

ĐH Tôn Đức Thắng

Nguyễn Huy Tuấn

Nguyễn Văn Chương

Nguyễn Thành Chung

Nguyễn Thị Hồng

$$
\text { ĐH Cần Thơ }
$$

ĐH Duy Tân

ĐH Phenikaa

ĐH Công nghệ Giao thông Vận tải

ĐH Phenikaa

Trần Huy Hùng

ĐH Công nghệ TP.HCM

ĐH Nguyễn Tất Thành

ĐH Tôn Đức Thắng

Viện Hàn lâm KH\&CN Việt Nam

ĐH Tôn Đức Thắng

Nguyễn Nhật Tân

Nguyễn Minh Đức
175758

189665

155893

157490

157573

162070

162315

162396

164299

165970

167251

169046

169464

171457

172679

173185

173918

180201

185211

188289

189398

189853 
Như vậy, Việt Nam có tất cả 29 tổ chức có nhà nghiên cứu nội lực được vào danh sách trên, trong đó có 2 viện nghiên cứu, 1 học viện, 1 bệnh viện và còn lại là các nhà khoa học nội lực đến từ các đại học.

Tổ chức có nhiều nhà nghiên cứu nội lực nhất trong danh sách là trường ĐH Tôn Đức Thắng, có 9 người và chiếm $\mathbf{1 3 . 8 \%}$ trong tổng số trong cả nước. Các trường đại học ngoài công lập ĐH Duy Tân và ĐH Phenikaa tiếp tục có nhiều nhà nghiên cứu nội lực được liệt kê vào danh sách này, tương ứng có 7 và 6 người.

Các trường ĐH ở Đồng Bằng Sông Cửu Long và ở Miền Trung cũng có nhà khoa học nội lực trong danh sách này gồm ĐH Cần Thơ, ĐH Đồng Tháp và ĐH Quảng Bình.

Có thể có ý kiến cho rằng các nhà nghiên cứu được liệt kê vào danh sách thành tựu trọn đời thì đẳng cấp hơn những nhà nghiên cứu được liệt kê vào danh sách năm.

Tuy nhiên, việc so sánh hay xếp hạng thì lúc nào cũng dễ tranh cãi. Thực tế thì danh sách thành tựu trọn đời thường dành cho các nhà nghiên cứu lâu năm hơn, có trích dẫn tương đối ổn định.

Những nhà nghiên cứu trẻ thì ít khi được liệt kê vào danh sách này vì việc có trích dẫn khoa học cao trong thời gian ngắn nói chung là rất khó, có thể trừ những trường hợp hết sức ngoại lệ và đặc biệt xuất sắc.

Đối với danh sách thành tựu năm gần nhất, việc đánh giá trích dẫn khoa học được lấy trong năm gần nhất (theo định nghĩa) những có thể nói đó là thành tựu "chín mùi" của các nhà nghiên cứu; thực tế thì các nhà nghiên cứu này đã xây dựng nền tảng từ trước đó nhiều năm và để năm gần nhất có được thành tựu trích dẫn cao.

Tuy nhiên, qua thực tế của Việt Nam, có thể dễ thấy là việc được vào danh sách thành tựu trọn đời thì nói chung là khó hơn vào danh sách thành tựu năm gần nhất.

Những thành tựu trên cho thấy sự bứt phá của các đại học Việt Nam trong nghiên cứu khoa học và đặc biệt là trích dẫn khoa học. Việc này đã góp phần quan trọng vào quá trình nâng cao chất lượng nghiên cứu và giáo dục của đất nước, cũng như việc tăng cường sự nhận diện của thương hiệu Việt Nam/các tổ chức nghiên cứu của Việt Nam trên phạm vi toàn cầu. Và về phương diện xếp hạng đại học quốc tế thì trích dẫn khoa học là một tiêu chí rất quan trọng, có khi mang tính quyết định để các đại học Việt Nam được các tổ chức xếp hạng đại học thế giới ghi nhận.

Việc công trình được trích dẫn cao và được ứng dụng vào thực tiễn để phát triển thành các công nghệ/chính sách giúp ích cho cuộc sống hoặc có giá trị thương mại cao thông thường là hai khái niệm khác nhau.

Số lượng trích dẫn khoa học đến một cách tự nhiên thì nói chung là khó và khi đó thành tựu trích dẫn cao rất đáng được trân trọng nhưng việc này chỉ dừng ở khía cạnh nhận diện cho các công trình nghiên cứu, chứ việc được trích dẫn cao chưa phải là yếu tố quyết định cho giá trị đích thực của một công trình nghiên cứu như đã nêu. 
Ngoài ra, vẫn có các kỹ thuật để tăng trích dẫn khoa học và số lượng trích dẫn khoa học có được từ các kỹ thuật này thì có thể xem là trích dẫn ảo. Việc tăng trích dẫn khoa học theo hướng trích dẫn ảo thì không được ủng hộ.

Tóm lại, tình hình phát triển nghiên cứu khoa học của Việt Nam là khả quan trong thời gian qua. Việc các công trình nghiên cứu của Việt Nam được trích dẫn cao thì bước đầu là một sự nhận diện quan trọng trên phạm vi toàn cầu.

Thành tựu này chắc chắn là kết quả của những chính sách phù hợp, tiếp cận thông lệ quốc tế trong nghiên cứu khoa học của Việt Nam trong thời gian qua, trong đó có chính sách tự chủ cho các cơ sở nghiên cứu và giáo dục đại học của đất nước.

Bước tiếp theo chắc chắn là sẽ không chỉ dừng ở số lượng trích dẫn mà là việc đưa ngày càng nhiều các công trình nghiên cứu vào thực tiễn cuộc sống để Việt Nam có thể sở hữu thêm nhiều công nghệ, phát triển thêm nhiều chính sách và khi đó sản phẩm nghiên cứu sẽ đóng góp nhiều hơn nữa vào GDP của đất nước thông qua việc thương mại hóa các sản phẩm nghiên cứu.

\section{TS. Lê Văn Út, Trưởng nhóm Nhóm nghiên cứu trắc lượng thông tin, Trường Đại} học Tôn Đức Thắng

Link nội dung: https://dantri.com.vn/giao-duc-huong-nghiep/su-that-ve-noi-luc-vietnam-trong-trich-dan-khoa-hoc-hang-dau-the-gioi-2021-20211030083557165.htm 